\title{
Associação entre bruxismo e tempo de tela em adolescentes: estudo exploratório
}

\author{
Association between bruxism and screen time in adolescents: exploratory study \\ Asociación entre bruxismo y tempo de pantalla en adolescentes: estudio exploratorio
}

Recebido: 28/07/2021 | Revisado: 03/08/2021 | Aceito: 04/08/2021 | Publicado: 10/08/2021

\author{
Vanessa Rodrigues Monteiro \\ ORCID: https://orcid.org/0000-0003-1499-3245 \\ Universidade de Pernambuco, Brasil \\ E-mail: vanessa.monteiro@upe.br \\ Raíssa Soares dos Anjo \\ ORCID: https://orcid.org/0000-0002-4766-4272 \\ Universidade de Pernambuco, Brasil \\ E-mail: raissa_soares@hotmail.com \\ Letícia Fernanda Serafim Cabral \\ ORCID: https://orcid.org/0000-0001-7590-0917 \\ Universidade de Pernambuco, Brasil \\ E-mail: leticiafscabral@hotmail.com \\ Valdenice Aparecida de Menezes \\ ORCID: https://orcid.org/0000-0003-4183-3239 \\ Universidade de Pernambuco, Brasil \\ E-mail: valdmenezes@ hotmail.com \\ Viviane Colares \\ ORCID: https://orcid.org/0000-0003-2912-2100 \\ Universidade de Pernambuco, Brasil \\ E-mail: viviane.colares@upe.br \\ Carolina da Franca \\ ORCID: https://orcid.org/0000-0002-7365-2806 \\ Universidade de Pernambuco, Brasil \\ E-mail: carolina.franca@upe.br \\ Fabiana Godoy \\ ORCID: https://orcid.org/0000-0002-1946-9605 \\ Universidade de Pernambuco, Brasil \\ E-mail: Fabiana.godoy@upe.br
}

\begin{abstract}
Resumo
Os adolescentes fazem uso constante de tecnologias o que pode acarretar o excesso de uso de tela. Essas por sua vez geram consequências mentais, como estresse e ansiedade, que são fatores desencadeadores do bruxismo. Considerando isto, este estudo transversal objetivou analisar a associação entre bruxismo e tempo de tela em 236 adolescentes, com faixa etária entre 14 e 19 anos, de ambos os sexos, estudantes de ensino médio de escolas públicas. Os dados foram coletados através de questões contidas na Pesquisa Nacional de Saúde do Escolar (PeNSE), Youth Risk Behavior Survey (YRBS) e Critérios Diagnósticos de Pesquisa em Disfunção Temporomandibular (RDC/TMD). Foi usado o teste Qui-Quadrado e calculada a Razão de Prevalência (RP). Dentre os pesquisados 30,9\% eram bruxistas e $62,3 \%$ faziam uso excessivo de telas. Quanto ao bruxismo, não houve associação com as variáveis independentes estudadas. Já em relação ao tempo de tela, "Renda familiar mensal" mostrou associação (p-valor de 0,008), em que 76,1\% dos pesquisados que faziam uso de telas por mais de duas horas ao dia possuíam renda mensal entre um e dois salários-mínimos. Assim, não se constatou associação entre bruxismo e tempo de tela nos adolescentes. Bruxismo e tempo de tela também não foram associados as variáveis sexo e idade, entretanto, verificouse associação entre tempo de tela e renda familiar.
\end{abstract}

Palavras-chave: Adolescente; Atitude frente aos computadores; Bruxismo; Saúde mental; Tempo de tela.

\begin{abstract}
Adolescents make constant use of technologies, which can lead to overuse of the screen. These in turn generate mental consequences, such as stress and anxiety, which are triggering factors of bruxism. Considering this, this crosssectional study aimed to analyze the association between bruxism and screen time in 236 adolescents, aged between 14 and 19 years of both sexes, high school students from public schools. Data were collected through questions contained in the National School Health Survey (PeNSE), Youth Risk Behavior Survey (YRBS) and Diagnostic Criteria for Research in Temporomandibular Dysfunction (RDC/TMD). The Chi-Square test was used, and the Prevalence Ratio (PR) was calculated. Among the surveyed, 30.9\% were bruxists and $62.3 \%$ used screens excessively. As for bruxism, there was no association with the independent variables studied. In relation to screen time, "Monthly family income" showed an association (p-value of 0.008 ), in which $76.1 \%$ of the surveyed who used
\end{abstract}


screens for more than two hours a day had a monthly income between one and two minimum wages. Thus, there was no association between bruxism and screen time in adolescents. Bruxism and screen time were also not associated with the variables gender and age, however, there was an association between screen time and family income.

Keywords: Adolescent; Attitude towards computers; Bruxism; Mental health; Screen time.

\section{Resumen}

Los adolescentes hacen un uso constante de las tecnologías, lo que puede conducir a un uso excesivo de la pantalla. Estos a su vez generan consecuencias mentales, como el estrés y la ansiedad, que son factores desencadenantes del bruxismo. Teniendo en cuenta esto, este estudio transversal tuvo como objetivo analizar la asociación entre el bruxismo y el tiempo de pantalla en 236 adolescentes, con edades entre 14 y 19 años, de ambos sexos, estudiantes de secundaria de escuelas públicas. Los datos fueron recolectados a través de preguntas contenidas en la Encuesta Nacional de Salud Escolar (PeNSE), la Encuesta de Conducta de Riesgo juvenil (YRBS) y los Criterios diagnósticos para la investigación en disfunción temporomandibular (RDC/TMD). Se utilizó la prueba de Chi-Cuadrado y se calculó la Razón de Prevalencia (RP). Entre los encuestados, el 30,9\% eran de bruxistas y el 62,3\% utilizaban las pantallas en exceso. En cuanto al bruxismo, no hubo asociación con las variables independientes estudiadas. Con relación al tiempo de pantalla, "Ingreso familiar mensual" mostró una asociación (p-valor de 0,008), en la que 76,1\% de los encuestados que utilizaron pantallas por más de dos horas diarias tenían un ingreso mensual entre uno y dos salarios mínimos. Por lo tanto, no había asociación entre el bruxismo y el tiempo de pantalla en adolescentes. El bruxismo y el tiempo de pantalla tampoco se asociaron con las variables sexo y edad, sin embargo, hubo una asociación entre el tiempo de pantalla y los ingresos familiares.

Palabras clave: Adolescente; Actitud hacia las computadoras; Bruxismo; Salud mental; Tiempo de pantalla.

\section{Introdução}

A geração de indivíduos que têm acesso a computadores e tecnologia digital desde seu nascimento são chamados nativos digitais (Zimerman, 2012). Jovens imersos na cultura das novas mídias as consideram como parte integral de seu cotidiano e as utilizam de maneira diferencial quando comparada às gerações anteriores (Ferreira \& Sales, 2019).

Mídias como televisão, YouTube, filmes, jogos de vídeo game, ouvir música e navegar na internet estão inseridas no dia a dia e se tornaram essenciais na vida dos adolescentes (Wallace, 2015). A Academia Americana de Pediatria ("Children, Adolescents, and Television", 2001) recomendou limitar o tempo total de mídia até duas horas de "programação de qualidade" por dia. Em contrassenso, Hu et al. (2018) citou que uso de aparelhos eletrônicos por até três horas por dia é considerado normal nesta faixa etária. Já a literatura aponta que, na verdade, o uso de telas pelos adolescentes tem média de seis horas por dia (Wallace, 2015). E que oito em cada dez adolescentes excedem o tempo de tela recomendado (Lucena et al., 2015).

O uso de dispositivos de tela - smartphone, tablets, computadores - acarreta vários malefícios ao adolescente. Dentre estes, podem-se citar o risco de obesidade por meio de diversos mecanismos (Zeeni, et al., 2018), alterações posturais (K. Hansraj et al., 2014), desordens musculoesqueléticas (Brink et al., 2014), qualidade do sono (Kann et al., 2017) e problemas a saúde mental, como irritabilidade, ansiedade e dificuldade nas relações pessoais (Rubens et al., 2017), fatores estes que são considerados como possíveis causas do bruxismo.

O bruxismo deve ser considerado como o ato parafuncional caracterizado por ranger ou apertar os dentes de forma voluntária e/ou involuntariamente, ocorrendo durante o período diurno e/ou noturno, podendo causar danos ao sistema estomatognático (Rios et al., 2018), tendo entre eles, estresse e ansiedade como uma das suas possíveis causas (Oliveira et al., 2015). Além de ser associado a dor muscular, (Costa et al., 2017) e a má higiene do sono (Alencar et al., 2020). Pode ainda ser uma condição associada com injúrias às estruturas orofaciais e impacto negativo na qualidade de vida dos escolares (Carvalho et al., 2015).

Alguns bruxistas apertam os dentes enquanto estão acordados (bruxismo de vigília) e outros durante o sono (bruxismo do sono) ou combinado (Antunes et al., 2015). Devido ao estresse do dia a dia ser cada vez mais percebido em crianças e adolescestes, torna-se necessário a detecção precoce no intuito de impedir à instalação de males ainda mais prejudiciais e o comprometimento da qualidade de vida do indivíduo (Strausz et al., 2010). 
É crescente o número de adolescentes acometidos por bruxismo ao longo do tempo. Isso pode estar relacionado as mudanças no estilo de vida desses indivíduos, inclusive, a intensificação do uso de aparelhos eletrônicos. Nesse contexto, o estudo objetivou avaliar a associação entre bruxismo e tempo de tela em adolescentes que frequentam a escola.

\section{Metodologia}

Trata-se de um estudo exploratório transversal cuja amostra foi composta por adolescentes escolares, de ambos os sexos, na faixa etária de 14 a 19 anos, matriculados na rede estadual de ensino de Olinda, Região Metropolitana do Recife/PE. Vinte e sete escolas foram consideradas elegíveis para a aplicação do questionário, das quais seis foram selecionadas por meio de sorteio. Utilizaram-se os dados coletados dos primeiros 236 adolescentes pesquisados.

Os Termos de Consentimento Livre e Esclarecido (TCLE) foram fornecidos aos pais /responsáveis ou aqueles com mais de 18 anos para que estes assinassem, aceitando participar da pesquisa. Enquanto os Termos de Assentimento Livre e Esclarecido (TALE) foram entregues aos adolescentes menores de 18 anos para que estes assinassem, aceitando participar da pesquisa. Foram excluídos da pesquisa os adolescentes que apresentassem alguma deficiência ou disfunção que impossibilitasse o autopreenchimento do questionário.

A coleta dos dados foi feita por meio da aplicação de três questionários. Aplicou-se o instrumento utilizado na Pesquisa Nacional de Saúde do Escolar (PeNSE) para obtenção de dados sociodemográficos (sexo, idade, série, renda mensal, escolaridade da mães/do pai) nos quesitos 1, 8,10, 11 e 12; Youth Risk Behavior Survey (YRBS) no qual foram relevantes as questões 100 e 101 sobre tempo de tela, sendo preconizado como excesso de tempo de tela mais que duas horas por dia (AAP, 2001); e os dados relativos à presença do possível bruxismo, coletados por meio das perguntas 115 a 117 contidas no Critérios Diagnósticos de Pesquisa em Disfunção Temporomandibular (RDC/TMD), desenvolvido por Dworkin e Le Resche em 1992 e validado no Brasil por Lucena, Kosminsky, Costa e Góes (2006). O diagnóstico positivo para possível bruxismo foi obtido se o paciente relatasse que rangia ou apertava os dentes e apresentasse um ou mais dos três critérios mencionados no instrumento (AASM, 2005).

Os dados foram digitados em dupla entrada no software Epidata 3.3 e os erros encontrados na validação foram corrigidos, no período de julho de 2018. Em 2020, para análise de dados foram utilizados os Softwares STATA 12.0 para Windows, EPI INFO 7.2.2.16 e o Excel 2010. Para avaliar o perfil dos alunos foram calculadas as frequências absoluta e relativa. Foi aplicado o teste Qui-Quadrado para verificar a existência de associação para as variáveis categóricas. A Razão de Prevalência (RP) foi calculada com intervalo de confiança de $95 \%$. Todas as conclusões foram tiradas considerando o nível de significância de $5 \%$.

A pesquisa foi desenvolvida de acordo com a resolução no 466/2012, do conselho nacional da saúde do ministério da saúde onde foi submetido e aprovado no Comitê de Ética em Pesquisa da Universidade de Pernambuco, CAAE: 76609817.1.0000.5207.

\section{Resultados}

As características gerais dos adolescentes incluídos no presente estudo estão apresentadas na Tabela 1. Na amostra, $30,9 \%$ dos escolares eram possíveis bruxistas, $62,3 \%$ faziam uso excessivo de telas digitais, 50,4\% estavam na faixa etária em 14-16 anos de idade, 56,1\% eram do sexo feminino, 58,4\% das mães possuíam menos de 11 anos de anos de escolaridade, 59,3\% dos pais possuíam menos de 11 anos de escolaridade e 55,5\% tinham renda mensal de até um salário-mínimo. 
Tabela 1 - Dados gerais da amostra.

\begin{tabular}{|c|c|c|}
\hline Variáveis & $\mathbf{N}$ & $\%$ \\
\hline \multicolumn{3}{|l|}{ Possível Bruxismo } \\
\hline Sim & 73 & 30,9 \\
\hline Não & 163 & 69,1 \\
\hline \multicolumn{3}{|l|}{ Tempo de Tela (mais de $2 \mathrm{~h}$ ) } \\
\hline Sim & 147 & 62,3 \\
\hline Não & 89 & 37,7 \\
\hline \multicolumn{3}{|l|}{ Idade } \\
\hline $14-16$ & 119 & 50,4 \\
\hline $17-19$ & 117 & 49,6 \\
\hline \multicolumn{3}{|l|}{ Sexo } \\
\hline Feminino & 128 & 56,1 \\
\hline Masculino & 100 & 43,9 \\
\hline \multicolumn{3}{|l|}{ Série } \\
\hline Primeiro ano do ensino médio & 86 & 37,1 \\
\hline Segundo ano do ensino médio & 64 & 27,6 \\
\hline Terceiro ano do ensino médio & 82 & 35,3 \\
\hline \multicolumn{3}{|l|}{ Religião } \\
\hline Ter Religião & 158 & 67,2 \\
\hline Não ter Religião & 77 & 32,8 \\
\hline \multicolumn{3}{|l|}{ Escolaridade da Mãe } \\
\hline$<11$ anos & 115 & 58,4 \\
\hline 11 ou mais anos & 82 & 41,6 \\
\hline \multicolumn{3}{|l|}{ Escolaridade do Pai } \\
\hline$<11$ anos & 83 & 59,3 \\
\hline 11 ou mais anos & 57 & 40,7 \\
\hline \multicolumn{3}{|l|}{ Bolsa Família } \\
\hline Sim & 107 & 45,9 \\
\hline Não & 126 & 54,1 \\
\hline \multicolumn{3}{|l|}{ Renda familiar mensal } \\
\hline Até 1 salário-mínimo & 86 & 55,5 \\
\hline De 1 a 2 salários-mínimos & 46 & 29,7 \\
\hline Mais de 2 salários-mínimos & 23 & 14,8 \\
\hline
\end{tabular}

Fonte: Autores (2021).

A Tabela 2 apresenta as associações entre o possível bruxismo e as variáveis independentes da pesquisa. Dentre as estudadas, não houve associação estatisticamente significativa. 
Tabela 2 - Análise bivariada correspondente à associação entre possível bruxismo e variáveis independentes.

\begin{tabular}{|c|c|c|c|}
\hline \multirow[b]{2}{*}{ Variáveis } & \multicolumn{2}{|c|}{ Possível Bruxismo } & \multirow[b]{2}{*}{ p-valor $*$} \\
\hline & $\begin{array}{c}\text { Sim } \\
\mathbf{n}(\%)\end{array}$ & $\begin{array}{c}\text { Não } \\
\text { n (\%) }\end{array}$ & \\
\hline \multicolumn{4}{|l|}{ Tempo de Tela (mais de $2 \mathrm{~h}$ ) } \\
\hline Sim & $47(32,0)$ & $100(68,0)$ & 0,657 \\
\hline Não & $26(29,2)$ & $63(70,8)$ & \\
\hline \multicolumn{4}{|l|}{ Idade } \\
\hline $14-16$ & $39(32,8)$ & $80(67,2)$ & 0,537 \\
\hline $17-19$ & $34(29,1)$ & $83(70,9)$ & \\
\hline \multicolumn{4}{|l|}{ Sexo } \\
\hline Feminino & $40(31,3)$ & $88(68,7)$ & 0,968 \\
\hline Masculino & $31(31,0)$ & $69(69,0)$ & \\
\hline \multicolumn{4}{|l|}{ Série } \\
\hline Primeiro ano do ensino médio & $27(31,4)$ & $59(68,6)$ & 0,111 \\
\hline Segundo ano do ensino médio & $26(40,6)$ & $38(59,4)$ & \\
\hline Terceiro ano do ensino médio & $20(24,4)$ & $62(75,6)$ & \\
\hline \multicolumn{4}{|l|}{ Religião } \\
\hline Ter Religião & $49(31,0)$ & $109(69,0)$ & 0,981 \\
\hline Não ter Religião & $24(31,2)$ & $53(68,8)$ & \\
\hline \multicolumn{4}{|l|}{ Escolaridade da Mãe } \\
\hline$<11$ anos & $35(30,4)$ & $80(69,6)$ & 0,944 \\
\hline 11 ou mais anos & $25(30,5)$ & $57(69,5)$ & \\
\hline \multicolumn{4}{|l|}{ Escolaridade do Pai } \\
\hline$<11$ anos & $25(30,1)$ & $58(69,9)$ & 0,297 \\
\hline 11 ou mais anos & $22(38,6)$ & $35(61,4)$ & \\
\hline \multicolumn{4}{|l|}{ Bolsa Família } \\
\hline Sim & $32(29,9)$ & $75(70,1)$ & 0,863 \\
\hline Não & $39(31,0)$ & $87(69,0)$ & \\
\hline \multicolumn{4}{|l|}{ Renda familiar mensal } \\
\hline Até 1 salário-mínimo & $24(27,9)$ & $62(72,1)$ & 0,285 \\
\hline De 1 a 2 salários-mínimos & $12(26,1)$ & $34(73,9)$ & \\
\hline Mais de 2 salários-mínimos & $10(43,5)$ & $13(56,5)$ & \\
\hline
\end{tabular}

(*) Teste Qui-Quadrado. Fonte: Autores (2021).

A Tabela 3 apresenta as associações entre o tempo de tela e as variáveis independentes da pesquisa. Dentre as variáveis independentes estudadas, apenas "Renda familiar mensal" teve associação com o tempo de tela (p-valor de 0,008$)$ e $76,1 \%$ dos pesquisados que faziam uso de telas por mais de duas horas ao dia possuíam renda entre um e dois salários-mínimos por mês. O que traz o entendimento que renda familiar mensal superior a um salário-mínimo gera uma maior chance de excesso de tempo de tela. 
Tabela 3 - Análise bivariada correspondente à associação entre tempo de tela e variáveis independentes.

\begin{tabular}{|c|c|c|c|}
\hline \multirow[b]{2}{*}{ Variáveis } & \multicolumn{2}{|c|}{ Tempo de Tela (mais de 2h) } & \multirow[b]{2}{*}{ p-valor * } \\
\hline & $\begin{array}{c}\text { Sim } \\
\text { n (\%) }\end{array}$ & $\begin{array}{c}\text { Não } \\
\text { n (\%) }\end{array}$ & \\
\hline \multicolumn{4}{|l|}{ Idade } \\
\hline $14-16$ & $76(63,9)$ & $43(36,1)$ & 0,614 \\
\hline $17-19$ & $71(60,7)$ & $46(39,3)$ & \\
\hline \multicolumn{4}{|l|}{ Sexo } \\
\hline Feminino & $79(61,7)$ & $49(38,3)$ & 0,505 \\
\hline Masculino & $66(66,0)$ & $34(34,0)$ & \\
\hline \multicolumn{4}{|l|}{ Série } \\
\hline Primeiro ano do ensino médio & $59(68,6)$ & $27(31,4)$ & 0,160 \\
\hline Segundo ano do ensino médio & $42(65,6)$ & $22(34,4)$ & \\
\hline Terceiro ano do ensino médio & $45(54,9)$ & $37(45,1)$ & \\
\hline \multicolumn{4}{|l|}{ Religião } \\
\hline Ter Religião & $99(62,7)$ & $59(37,3)$ & 0,962 \\
\hline Não ter Religião & $48(62,3)$ & $29(37,7)$ & \\
\hline \multicolumn{4}{|l|}{ Bolsa Família } \\
\hline Sim & $62(57,9)$ & $45(42,1)$ & 0,213 \\
\hline Não & $83(65,9)$ & $43(34,1)$ & \\
\hline \multicolumn{4}{|l|}{ Escolaridade da Mãe } \\
\hline$<11$ anos & $74(64,3)$ & $41(35,7)$ & 0,318 \\
\hline 11 ou mais anos & $47(57,3)$ & $35(42,7)$ & \\
\hline \multicolumn{4}{|l|}{ Escolaridade do Pai } \\
\hline$<11$ anos & $52(62,7)$ & $31(37,3)$ & 0,881 \\
\hline 11 ou mais anos & $35(61,4)$ & $22(38,6)$ & \\
\hline \multicolumn{4}{|l|}{ Renda familiar mensal } \\
\hline Até 1 salário-mínimo & $44(51,2)$ & $42(48,8)$ & 0,008 \\
\hline De 1 a 2 salários-mínimos & $35(76,1)$ & $11(23,9)$ & \\
\hline Mais de 2 salários-mínimos & $17(73,9)$ & $6(26,1)$ & \\
\hline
\end{tabular}

(*) Teste Qui-Quadrado. Fonte: Autores (2021).

\section{Discussão}

\subsection{Bruxismo}

A prevalência de possível bruxismo no presente estudo foi considerada semelhante quando comparada a outros também realizados em adolescentes. Souza et al. (2018) apresentaram prevalência de 22,2\%, Arman et al. (2016) de 24,5\% e Carra et al. (2011) 15\%, salientando que entre esses estudos supracitados não houve distinção entre tipos de bruxismo. Nota-se que, quando há a diferenciação entre o bruxismo do sono e o de vigília, a prevalência de bruxismo se torna menor em comparação aos estudos em que isso não foi feito. Em Emodi Perlman et al. (2016) a prevalência do bruxismo do sono foi 9,2\% e de vigília 19,2\%, enquanto Van Selms et al. (2012) descreveu 14,8\% para o do sono e 8,7\% para o de vigília. A prevalência de bruxismo na população estudada pode se justificar pelo fato de ser o possível bruxismo, em que o instrumento de diagnóstico é distinto das demais pesquisas citadas, a amostra em questão tem características sociodemográficas distintas, como faixa etária, além de não especificar o tipo de bruxismo entre os acometidos. 
Em relação ao sexo, este estudo não apresenta diferença significativa entre os bruxistas, o que corrobora os estudos de Emodi Perlman et al. (2016), Arman et al. (2016) e Turkoglu et al. (2013). Já Van Selms et al. (2012) e Strausz et al. (2010) afirmaram predominância ao ranger os dentes do sexo feminino. Em contraponto, Souza et al. (2018) relataram prevalência do sexo masculino, associando ao fato de os meninos serem mais agitados e, culturalmente, motivados a conter suas emoções, o que poderia favorecer a ocorrência dos movimentos involuntários.

Este estudo foi dividido em dois grupos de faixa etária, em que prevalência do possível bruxismo se encontra no grupo dos mais jovens, todavia, não é significativa. Emodi Perlman et al. (2016) dividiram em faixa etárias entre 12-15 anos e 16-18 anos e, no bruxismo de vigília, encontraram o mesmo resultado. O que pode ser justificado pelos grupos, das duas pesquisas, serem divididos de maneira semelhante. Van Selms et al. (2012) e Souza et al. (2018) não dividiram em grupos de faixa etária, fizeram a prevalência de cada idade. A faixa etária geral de Souza et al. (2018) era entre 11 e 14 anos, sendo maior o bruxismo encontrado nos adolescentes de 12 e 14 anos com 25,5\%. Carra et al. (2011) dividiu sua faixa etária geral em duas, sendo de 7-12 anos e 13-17 anos, e, notou menor frequência de bruxismo em indivíduos maiores de 12 anos. Isso pode ser justificado pelo fato dos pais a partir dessa idade visitarem menos seus filhos durante o sono. As divergências encontradas entre os resultados podem se dar pelos métodos de estudo e faixas-etárias distintas.

No que se refere a escolaridade dos pais, neste estudo, mais da metade dos pais estudaram menos que 11 anos, ou seja, não completaram o período escolar. Dentre os estudos mais recentes sobre o tema, apenas Souza et al. (2018) analisaram esta variável e encontraram que 38,7\% das mães estudaram até sete anos e 46,5\% delas estudaram entre sete e 11 anos. Em concordância, ambos os estudos brasileiros retratam que o grau de escolaridade familiar é inferior a 11 anos. Quanto ao grau de escolaridade das mães e o percentual de filhos bruxistas, encontrou-se prevalência foi semelhante; já entre os pais, houve uma pequena diferença não significante. Os demais autores não avaliaram a escolaridade dos pais, com isso, esse tópico não pode ser adequadamente debatido.

Neste estudo, a maior concentração de indivíduos com bruxismo foi encontrada entre os que recebiam mais que dois salários-mínimos por mês. Entretanto, não se observou associação estatisticamente significante, corroborando com os achados de Souza et al. (2018), em que a renda familiar não influenciou na presença de bruxismo.

\subsection{Tempo de tela}

Nesse estudo, a prevalência do tempo de tela maior que duas horas entre os adolescentes foi semelhante ao estudo de Atkin et al. (2014), em que pelo menos 2/3 dos participantes $(66,6 \%)$ ultrapassaram duas horas/dia de tempo de tela. Já Tenório et al. (2010) dividiram as prevalências em dias de semana e final de semana, sendo 40,9\% e 49,9\%, respectivamente, e Cao et al. (2011) definiram prevalência de 26,1\% de alta exposição de tela/dia dos adolescentes, o que resulta em prevalência menor que a encontrada no atual estudo. Por outro lado, ainda se apontou valor superior ao atual na pesquisa de Sousa e Silva (2017), em que foi relatado que a prevalência de uso excessivo de tela foi de $86,37 \%$; desse tempo, o uso de computador foi maior com 55,24\%, seguido de televisão 51,56\% e videogame 15,35\%. Atkin et al. (2014), Cao et al. (2011), Ramos--Jimenez et al. (2013) e Smith-Menezes et al. (2013) consideraram mais do que duas horas de tela por dia como excesso de uso. Já Tenório et al. (2010) consideram o valor de três horas ou mais por dia. Sousa \& Silva (2017) em sua pesquisa, não citam exatamente a quantidade de horas, mas levaram em consideração o tempo de tela recomendado; subentendendo-se que foi a partir de duas horas/dia tido como tempo excedido. Assim, a diferença de prevalências pode ser justificada pelas faixas etárias distintas, diferentes critérios de uso das telas e ainda método de estudo.

Quanto ao sexo, este estudo mostra que não houve diferença estatisticamente significante, assim como, Rey-Lopes (2011) que encontrou que assistindo televisão, o tempo de tela é similar entre os sexos. Por outro lado, o dado do sexo masculino ser prevalente, foi defendido por Tenório et al. (2010) quando dizem que exposição ao tempo de tela nos fins de 
semana foi maior entre os rapazes; por Rey-Lopes (2011), quando diz que meninos gastam mais tempo usando computador e videogames; por Babey et al. (2013); e, ainda Atkin et al. (2014) apresentaram que meninas tiveram menor probabilidade de exceder 2 horas/dia de tempo de tela. Em contrapartida, Feng et al. (2014) encontraram que 74,4\% das adolescentes, do seu estudo, tiveram tempo de tela $>2 \mathrm{~h} /$ dia, enquanto os adolescentes do sexo masculino tiveram $71,2 \%$. Sousa \& Silva (2017) afirmaram que as meninas possuem maior chance de assistir televisão em excesso, mas que os meninos possuem tempo de videogame e de tela total maiores. Essas discrepâncias podem ser justificadas pelo fato de a atual pesquisa não estudar as telas utilizadas separadamente; o método dos estudos é divergente.

Em relação a faixa etária, nessa pesquisa, não houve associação estatisticamente significativa. Sousa \& Silva (2017) tiveram divisão semelhante, a faixa etária de 14-16 com prevalência de 85,53\%, e a de 17-19 com 78,2\%, e perceberam que nos adolescentes mais jovens existe maior chance de o tempo total de tela ser acima do recomendado. Do mesmo modo, Tenório et al. (2010) encontraram prevalência de 67,5\% entre 14 e 16 anos e 63,4\% nos de 17 a 19 anos, e concluíram que os mais velhos e que estudam a noite apresentam menor chance de tempo excessivo de tela. Esses resultados ratificam o presente estudo em que a prevalência de uso do tempo de tela entre os mais jovens é maior. Rey-Lopes et al (2011) pesquisaram em adolescentes entre 13 e 18,5 anos, e afirmou que garotas com idade de 13 anos estão mais sugestivas a exceder tempo de tela de videogames que as garotas mais velhas. Atkin et al. (2014) não encontraram associação significante entre essas variáveis. Maras et al. (2015) afirmaram que existe relação entre faixa etária e tempo de tela, mas não desenvolvem em seu estudo.

Nesse estudo foi prevalente tempo excessivo de tela entre os adolescentes cujas mães possuíam escolaridade menor que 11 anos, todavia essa associação não foi significante. Do mesmo modo, Babey et al. (2013) correlacionam tempo dedicado à televisão ao menor nível educacional dos pais; e Atkin et al. (2014) também encontram esse resultado, porém não especificam em que atividades esse tempo de tela foi gasto. Rey-Lopes et al. (2011) afirmam que adolescentes cujos pais possuem ensino superior completo assistem menos televisão, enquanto aqueles que os pais que concluíram somente a escola assistem mais televisão, usam mais computador e videogames. Também para Sousa \& Silva (2017), os adolescentes que as mães estudaram menos de oito anos tiveram mais chances de assistirem televisão em excesso. Ou seja, apesar da pequena diferença, quanto maior a educação dos pais menor o tempo de tela diário. Todos os autores ratificando o resultado encontrado na atual pesquisa.

Acerca da renda familiar mensal, este estudo encontrou associação ao uso excessivo de tempo de tela ( $\mathrm{p}$ valor $=$ 0,008). A prevalência maior foi entre as famílias com renda maior que um e dois salários-mínimos. Em compensação, Sousa \& Silva (2017) afirmaram que aqueles com baixo nível econômico tiveram maior chance de tempo total de tela excessivo. SmithMenezes et al. (2013) também relaciona o uso de computador, televisão e videogame em excesso aos adolescentes de baixo nível econômico, assim como, Babey et al. (2013) que relacionou o tempo gasto assistindo televisão à menor renda familiar. Esses resultados podem ser justificados pelos diferentes critérios de tempo de tela utilizados; Smith-Menezes et al. (2013) e Babey et al. (2013) citam seus usos de tela, e não incluem smartphone, por exemplo, ainda usam diferentes métodos de estudo e características sociodemográficas. Além de que, a população estudada, por mais que tenha renda maior que dois saláriosmínimos mensal, esse valor ainda é considerado baixo.

\subsection{Associação entre bruxismo e tempo de tela}

Observou-se que $32 \%$ dos participantes apresentavam possível bruxismo e também faziam uso de tela por mais de duas horas por dia, todavia esse resultado não foi estatisticamente significante $(0,657)$. Esse tópico não foi debatido por ser o primeiro na literatura a tratar desse tema de maneira direta.

Quanto as limitações desse estudo, o desenho transversal surge como uma pois não é possível estabelecer uma relação de causa e efeito. Além disso, essa pesquisa assim como outras sobre tempo de tela em adolescentes, considerou como tempo 
limite de uso de telas diários duas horas por dia preconizado. Entretanto, o quantitativo foi estabelecido pela Academia Americana de Pediatria há mais de 20 anos e não corresponde à realidade atual. Outro aspecto relevante é que a instituição apenas considera televisão para avaliar o tempo de tela, e diversos meios de tela fixas e portáteis existem, o que possivelmente aumentaria o tempo de uso diário nos estudos sobre o tema. Deve-se salientar também que os dados utilizados nessa pesquisa foram coletados em 2018, e com o surgimento da pandemia de COVID-19, as atividades escolares e recreativas se restringiram ao uso de eletrônicos diante do isolamento social, situação também não ponderada pela Academia Americana de Pediatria.

\section{Considerações Finais}

Nesse estudo, não foi observada associação entre bruxismo e o tempo de tela em adolescentes que frequentam a escola. Em relação a prevalência do bruxismo e tempo de tela, não houve diferença em relação ao sexo e idade, escolaridade materna e paterna. Porém o excesso de tempo de tela foi maior entre aqueles com renda familiar mensal maior que um saláriomínimo.

Recomenda-se a realização de novos estudos com amostra maior, desenhos longitudinais e de intervenção. Outra possibilidade, seria reavaliar a população estudada nessa pesquisa e comparar os resultados obtidos diante das alterações temporais, principalmente, a pandemia de COVID-19. Além disso, a atualização da quantidade de horas destinadas ao uso de telas diariamente e incorporação dos outros dispositivos eletrônicos além da televisão. Ressalta-se que diante do exposto esse período diário seja irrisório para maioria dos adolescentes, o que pode justificar a não-associação significativa com o bruxismo.

\section{Referências}

Alencar, T. C., Batista, J. F. D. O. L., Tolêdo, J. M. G. F., da Silveira, M. A. C., Lyra, M. C. A., Vieira, S. C. M., \& Heimer, M. V. (2020). Associação entre qualidade do sono, bruxismo do sono e sonolência diurna excessiva em adolescentes. Brazilian Journal of Development, 6(9), 69157-69173.

Antunes, L. A. A., Castilho, T., Marinho, M., Fraga, R. S., \& Antunes, L. S. (2015). Childhood bruxism: Related factors and impact on oral health-related quality of life. Special Care in Dentistry, 36(1), 7-12. https://doi.org/10.1111/scd.12140

Arman, K., Petruninaitè, A., Grigalauskienè, R., \& Slabšinskienė, E. (2016). Stress experience and effect on self-perceived oral health status among high school students. Stomatologija, 18(3), 75-79.

Atkin, A. J., Sharp, S. J., Corder, K., \& van Sluijs, E. M. F. (2014). Prevalence and Correlates of Screen Time in Youth. American Journal of Preventive Medicine, 47(6), 803-807. https://doi.org/10.1016/j.amepre.2014.07.043

Babey, S. H., Hastert, T. A., \& Wolstein, J. (2013). Adolescent sedentary behaviors: Correlates differ for television viewing and computer use. Journal of Adolescent Health, 52(1), 70-76. https://doi.org/10.1016/j.jadohealth.2012.05.001

Brink, Y., Louw, Q., Grimmer, K., \& Jordaan, E. (2014). The spinal posture of computing adolescents in a real-life setting. BMC Musculoskeletal Disorders, 15(1). https://doi.org/10.1186/1471-2474-15-212

Cao, H., Qian, Q., Weng, T., Yuan, C., Sun, Y., Wang, H., \& Tao, F. (2011). Screen time, physical activity and mental health among urban adolescents in China. Preventive Medicine, 53(4-5), 316-320. https://doi.org/10.1016/j.ypmed.2011.09.002

Carra, M. C., Huynh, N., Morton, P., Rompré, P. H., Papadakis, A., Remise, C., \& Lavigne, G. J. (2011). Prevalence and risk factors of sleep bruxism and wake-time tooth clenching in a 7- to 17-yr-old population. European Journal of Oral Sciences, 119(5), 386-394. https://doi.org/10.1111/j.16000722.2011.00846.x

Carvalho, A. d. M. B., Lima, M. d. D. M. d., Silva, J. M. N. d., Neta, N. B. D., \& Moura, L. d. F. A. d. D. (2015). Bruxismo e qualidade de vida em escolares de 11 a 14 anos. Ciência \& Saúde Coletiva, 20(11), 3385-3393. https://doi.org/10.1590/1413-812320152011.20772014

Children, Adolescents, and Television. (2001). PEDIATRICS, 107(2), 423-426. https://doi.org/10.1542/peds.107.2.423

Costa, A. R. O., de Oliveira, E. S., de Oliveira, D. W. D., Tavano, K. T. A., Murta, A. M. G., Gonçalves, P. F., \& Flecha, O. D. (2017). Prevalência e fatores associados ao bruxismo em universitários: um estudo transversal piloto. Revista Brasileira de Odontologia, 74(2), 120.

Emodi Perlman, A., Lobbezoo, F., Zar, A., Friedman Rubin, P., van Selms, M. K. A., \& Winocur, E. (2016). Self-Reported bruxism and associated factors in Israeli adolescents. Journal of Oral Rehabilitation, 43(6), 443-450. https://doi.org/10.1111/joor.12391

Feng, Q., Zhang, Q.-1., Du, Y., Ye, Y.-1., \& He, Q.-q. (2014). Associations of Physical Activity, Screen Time with Depression, Anxiety and Sleep Quality among Chinese College Freshmen. PLoS ONE, 9(6), Artigo e100914. https://doi.org/10.1371/journal.pone.0100914

Ferreira, A. G., \& Sales, S. R. (2019). " Nativos digitais"," geração internet"," Homo zappiens"," ciborgue": juventude conectada às tecnologias 
digitais. TEXTURA-Revista de Educação e Letras, 21(47).

Hu, E. Y., Ramachandran, S., Bhattacharya, K., \& Nunna, S. (2018). Obesity Among High School Students in the United States: Risk Factors and Their Population Attributable Fraction. Preventing Chronic Disease, 15. https://doi.org/10.5888/pcd15.180122

K Hansraj, K. (2014). Assessment of stresses in the cervical spine caused by posture and position of the head. Surg Technol Int, 25, $277-279$.

Kann, L., McManus, T., Harris, W. A., Shanklin, S. L., Flint, K. H., Queen, B., Lowry, R., Chyen, D., Whittle, L., Thornton, J., Lim, C., Bradford, D., Yamakawa, Y., Leon, M., Brener, N., \& Ethier, K. A. (2018). Youth risk behavior surveillance — united states, 2017. MMWR. Surveillance Summaries, 67(8), 1-114. https://doi.org/10.15585/mmwr.ss6708a1

Lucena, J. M. S. D., Cheng, L. A., Cavalcante, T. L. M., Silva, V. A. D., \& Farias, J. C. D. (2015). Prevalência de tempo excessivo de tela e fatores associados em adolescentes. Revista Paulista de Pediatria, 33, 407-414.

Maras, D., Flament, M. F., Murray, M., Buchholz, A., Henderson, K. A., Obeid, N., \& Goldfield, G. S. (2015). Screen time is associated with depression and anxiety in Canadian youth. Preventive Medicine, 73, 133-138. https://doi.org/10.1016/j.ypmed.2015.01.029

Oliveira, M. T. d., Bittencourt, S. T., Marcon, K., Destro, S., \& Pereira, J. R. (2015). Sleep bruxism and anxiety level in children. Brazilian Oral Research, 29(1), 1-5. https://doi.org/10.1590/1807-3107bor-2015.vol29.0024

Ramos-Jimenez, A., Wall-Medrano, A., Esparza-Del Villar, O., \& Hernández-Torres, R. P. (2013). Design and validation of a self-administered test to assess bullying (bull-M) in high school Mexicans: a pilot study. BMC public health, 13(1), 1-7.

Rey-Lopez, J. P., Tomas, C., Vicente-Rodriguez, G., Gracia-Marco, L., Jimenez-Pavon, D., Perez-Llamas, F., Redondo, C., Bourdeaudhuij, I. D., Sjostrom, M., Marcos, A., Chillon, P., \& Moreno, L. A. (2010). Sedentary behaviours and socio-economic status in Spanish adolescents: The AVENA study. The European Journal of Public Health, 21(2), 151-157. https://doi.org/10.1093/eurpub/ckq035

Rios, L. T., Aguiar, V. N. P., Machado, F. C., Rocha, C. T., \& Neves, B. G. (2018). Bruxismo infantil e sua associação com fatores psicológicos - revisão sistemática da literatura. Revista de Odontologia da Universidade Cidade de São Paulo, 30(1), 64. https://doi.org/10.26843/ro_unicidv3012018p64-76

Rubens, S. L., Evans, S. C., Becker, S. P., Fite, P. J., \& Tountas, A. M. (2016). Self-Reported time in bed and sleep quality in association with internalizing and externalizing symptoms in school-age youth. Child Psychiatry \& Human Development, 48(3), 455-467. https://doi.org/10.1007/s10578-016-0672-1

Smith-Menezes, A., Duarte, M. d. F. d. S., \& Silva, R. J. d. S. (2012). Inatividade física, comportamento sedentário e excesso de peso corporal associados à condição socioeconômica em jovens. Revista Brasileira de Educação Física e Esporte, 26(3), 411-418. https://doi.org/10.1590/s1807-55092012000300007

Sousa, G. R. d., \& Silva, D. A. S. (2017). Sedentary behavior based on screen time: Prevalence and associated sociodemographic factors in adolescents. Ciência \& Saúde Coletiva, 22(12), 4061-4072. https://doi.org/10.1590/1413-812320172212.00472016

Souza, H. C. S., Lima, M. d. D. M. d., Dantas Neta, N. B., Tobias, R. Q., Moura, M. S. d., \& Moura, L. d. F. A. d. D. (2018). Prevalência e fatores associados ao bruxismo do sono em adolescentes de Teresina, Piauí. Revista Brasileira de Epidemiologia, 21. https://doi.org/10.1590/1980-549720180002

Strausz, T., Ahlberg, J., Lobbezoo, F., Restrepo, C. C., Hublin, C., Ahlberg, K., \& Könönen, M. (2010). Awareness of tooth grinding and clenching from adolescence to young adulthood: A nine-year follow-up. Journal of Oral Rehabilitation, 37(7), 497-500. https://doi.org/10.1111/j.1365-2842.2010.02071.X

Türkoğlu, S., Akça, Ö. F., Türkoğlu, G., \& Akça, M. (2013). Psychiatric disorders and symptoms in children and adolescents with sleep bruxism. Sleep and Breathing, 18(3), 649-654. https://doi.org/10.1007/s11325-013-0928-y

Van Selms, M. K. A., Visscher, C. M., Naeije, M., \& Lobbezoo, F. (2012). Bruxism and associated factors among Dutch adolescents. Community Dentistry and Oral Epidemiology, 41(4), 353-363. https://doi.org/10.1111/cdoe.12017

Wallace, K. (2015). Teens spend a 'mind-boggling' 9 hours a day using media, report says. CNN. com. November, 4. https://edition.cnn.com/2015/11/03/health/teens-tweens-media-screen-use-report/index.html

Zeeni, N., Doumit, R., Abi Kharma, J., \& Sanchez-Ruiz, M. J. (2018). Media, technology use, and attitudes: Associations with physical and mental well-being in youth with implications for evidence-based practice. Worldviews on Evidence-Based Nursing, 15(4), 304-312.

Zimerman, M. (2012). Digital natives, searching behavior and the library. New Library World, 113(3/4), 174-201. https://doi.org/10.1108/03074801211218552 